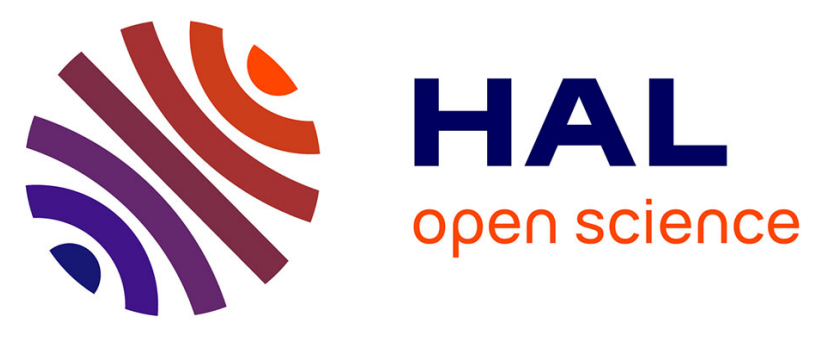

\title{
Technical note on the harvest of periosteal forearm composite free flaps in the treatment of early mandibular osteoradionecrotic injury
}

Jérémie Bettoni, Matthieu Olivetto, J. Bouaoud, Bernard Devauchelle

\section{- To cite this version:}

Jérémie Bettoni, Matthieu Olivetto, J. Bouaoud, Bernard Devauchelle. Technical note on the harvest of periosteal forearm composite free flaps in the treatment of early mandibular osteoradionecrotic injury. Journal of Stomatology, Oral and Maxillofacial Surgery, 2019, 120 (6), pp.570-572. 10.1016/j.jormas.2019.04.011 . hal-03048180

\section{HAL Id: hal-03048180 https: / hal.sorbonne-universite.fr/hal-03048180}

Submitted on 9 Dec 2020

HAL is a multi-disciplinary open access archive for the deposit and dissemination of scientific research documents, whether they are published or not. The documents may come from teaching and research institutions in France or abroad, or from public or private research centers.
L'archive ouverte pluridisciplinaire HAL, est destinée au dépôt et à la diffusion de documents scientifiques de niveau recherche, publiés ou non, émanant des établissements d'enseignement et de recherche français ou étrangers, des laboratoires publics ou privés. 
1 Title

12 3

Technical note on the harvest of periosteal forearm composite free flaps in the treatment of early mandibular osteoradionecrotic injury

Bettoni Jérémie $^{1,2}$, Olivetto Matthieu ${ }^{1,2}$, Bouaoud Jébrane ${ }^{3}$, Devauchelle Bernard ${ }^{1,2}$

${ }^{1}$ Department of Maxillofacial Surgery, University Hospital of Amiens

Avenue Laennec, 80000 Amiens, France

${ }^{2}$ EA CHIMERE, Picardie Jules Verne University

Avenue Laennec, 80000 Amiens, France

${ }^{3}$ Department of Oral and Maxillofacial Surgery, Sorbonne University, Pitié-Salpétrière Hospital Boulevard de l'Hôpital, 75013, Paris, France

\section{Corresponding author:}

Jérémie Bettoni, M.D.

Department of Maxillofacial Surgery, University Hospital of Amiens

Avenue Laennec, 80000 Amiens, France

+33 322089050 (Fax: +33 3226679 94)

jeremiebettoni@gmail.com 
Abstract (150 words)

26

Periosteal radial forearm free flaps allow functional and curative treatment of osteoradionecrotic jaw lesions. The flap responds physiologically to hypoxia, hypocellularity, and hypovascularity, which are characteristic results of osteoradionecrotic injury. Here, we propose a reproducible, simple, and safe method for harvesting a forearm free flap, allowing us to obtain a periosteal composite free flap. Our technique offers potential early management of osteoradionecrotic lesions resistant to medical treatment. Furthermore, the forearm periosteal composite free flap offers the advantage of a long vascular pedicle which is ideally suited for necks which have been submitted to irradiation or multiple operations. Thusly, vascular anastomosis can be performed at a distance from the irradiated areas. Compared to other periosteal free flaps, such as those harvested from the iliac crest or the internal femoral condyle, the presence of a skin paddle facilitates clinical flap monitoring, optimizes the restoration of bone sealing, and facilitates the treatment of scarring.

Keywords:

Osteoradionecrosis, periosteal free flap, management, revascularization surgery, forearm periosteal free flap 
Mandibular osteoradionecrosis (ORN) is a severe complication of radiotherapy characterized by hypoxic, hypovascular, and hypocellular bone ${ }^{1}$ which has lost its capacity to renew itself. Clinically, ORN presents as spontaneous mandibular bone exposure or occurs following an endobuccal surgical procedure. ${ }^{2}$ Different complications can occur, in particular cutaneous fistulas and/or pathological fractures. Therapeutic revascularization surgery (resection of the pathological bone with immediate reconstruction by microanastomosis of a flap) is the gold standard, with an efficacy rate of between $86 \%$ and $100 \%{ }^{3,4}$ By contrast, for treatment requiring a consensus, such as long-term antibiotic therapy or the Pentoclo protocol, efficacy assessment requires evaluation by randomized double-blind placebocontrolled studies. ${ }^{5}$ However, due to the difficulties of surgery in irradiated areas, the scarcity of vessels, and the high risk of failure, most teams consider it preferable to reserve radical management with free flap reconstruction for cases of ORN with fractures. ${ }^{6}$ Furthermore, malnutrition secondary to reactional trismus is a criterion of severity, complicating any surgical management. In 2009, D'Hauthuille et al. ${ }^{7}$ proposed the early management of ORN relating to the underlying risk of neoplasia and the tendency of the lesions to progress. This management is based on marginal bone resection with reconstruction by a free flap "cover" which preserves the food masticating function while revascularizing the underlying bone. Thus, periosteal free 
flaps (internal condyle, femoral, or others) are recommended for their osteoinductive and osteogenic properties. Indeed, the supply of vascularized living tissues within an irradiated environment constitutes a physiological response to the treatment of osteoradionecrosis. The efficacy rate of $100 \%$ confirms that it remains essential to design mandibular ORN curative surgery with a unique and functional approach.

From this perspective, and taking into account the scarring fibrosis of the post-radial endobuccal mucosa, the forearm free flap with a radial periosteal component offers several advantages responding to the constraints of early osteoradionecrotic lesion revascularization surgery: i) it provides a periosteal membrane, and ii) its fasciocutaneous flap facilitates clinical flap monitoring and optimizes buccal tightness with tension-free sutures. Here, we describe our harvesting technique, ensuring fast and safe dissection. 


\section{Technical Note}

After verifying the ulnar vascularization of the palmar arch using Allen's test, the dimensions of a fasciocutaneous paddle is defined, centered on the radial vascular pedicle. It is important to overestimate the volume of the cutaneous paddle needed relative to the bone exposure area to allow tension-free suturing during endobuccal insertion.

Using a pneumatic tourniquet, a curvilinear incision starting from the fold of the elbow to the upper point of the skin paddle is performed. Then, the ulnar and radial sides of the skin paddle should be carefully incised to include the cephalic vein. It is important to begin the dissection of the fasciocutaneous paddle by the radial side of the flap to protect the cutaneous branches of the radial nerve. Once dissected and reclined by placing a Gillies hook or a Faraboeuf spreader on the brachioradial muscle tendon (Figure 1), the radial periosteum is incised with the length corresponding to the slice of mandibular bone section to be "resurfaced". The periosteum is then gently roughened to avoid it dissociating from the radial vascular pedicle.

The ulnar surface of the flap is secondarily dissected up to the tendon of the long flexor muscle of the carpus, which is then retracted with a Gillies hook, allowing exposure of the lower end of the radius. This facilitates the access to the radial periosteum, which should be incised and scraped until it reaches the contralateral detachment zone. Once the entire periosteum is released, the distal end of the radial 
vascular pedicle is dissected, then ligated to counter-incise the radial periosteum and 103 release it over its entire length. The limit of the proximal portion of the radial 3 periosteum is defined according to the required length.

The remaining steps of harvesting are the same as the conventional procedures described by Yang, in $1981 .^{8}$ The careful dissection of the radial vascular pedicle is carried out with ligatures of all collaterals until a sufficient vascular pedicle length is obtained to allow for anastomosis (Figure 2). Finally, after releasing the pneumatic tourniquet, the radial accompanying veins (vena comitans) are ligated to check for the presence of cephalic venous return. If there is no cephalic venous drainage, the cephalic vein is sacrificed and a accompanying vein is used. Once weaned, the flap is washed with a heparinized saline solution to prevent the risk of distal microthromboses.

When setting the flap in its position, the radial periosteum is sutured prior to the mandibular periosteum to isolate the section of bone. Then, the skin paddle is sutured without tension to the adjacent mucosa by separate stitches of absorbable braided sutures. In principle, microsurgical anastomoses should be performed using separate stitches of non-absorbable non-braided sutures (9/0 or 10/0) always starting with the vessels of the flap to avoid any disassociation of the intima from the media of the irradiated vessels during the passage of the needle, which would increase the thrombogenic risk. ${ }^{9}$ 


\section{Discussion}

By reporting our harvesting technique for periosteal forearm free flaps, we provide a safe and accessible alternative management option for early ORN or ORN refractory to medical treatment. Initially proposed by Kelley et al. ${ }^{10}$ for the optimization of vascularization of maxillary bone grafts and by Roselli et al. ${ }^{11}$ as a cancer surgery option for marginal mandibulectomy, the periosteal forearm free flap has many advantages for surgery performed in the irradiated area.

First, known for its reliability, ${ }^{12}$ the forearm free flap offers the advantage of having a long vascular pedicle to respond to the difficulties of avascular necks encountered subsequent to radiotherapy or multiple surgeries. Indeed, this characteristic makes it possible to perform microsurgical anastomosis on vessels located outside the irradiation area, such as the temporal or transverse vessels.

In addition, the skin paddle of the periosteal forearm composite free flap facilitates the good inset of the flap as well as treatment of scar ridges responsible for ankyloglossia or a reduced buccal opening. This is not possible with other periosteal free flaps ${ }^{7}$ such as those from the iliac crest or internal femoral condyle that tend to retract.

Finally, the presence of a skin paddle makes it easier to monitor the vitality of the free flap due to the recoloring time which can help to signal the the need for vascular anastomosis revision. Nevertheless, unlike the external brachial free flap with humeral periosteum, the adipose volume of the fasciocutaneous component of the 
144 forearm composite free flap can require a second surgical procedure to reduce or $1_{2}^{1} 45$ eliminate excess tissue.

3

$1_{5}^{4} 46$ Regarding the technical procedures of revascularization surgery using periosteal 6 $1_{8}^{7} 47$ forearm free flaps, the recent controversy on the effectiveness of the main ${ }_{1148}$ conservative treatment ${ }^{5,13-15}$ requires deep reflection on the part of the entire scientific 11 


\section{Conclusion}

$1 \frac{1}{2} 2$ The reliability of the forearm free flap and its vascular pedicle length makes it a flap 3 153 of choice in irradiated neck surgery. The periosteal vascularized transfer provides a 6 154 physiological response to the physiopathogenesis of ORN while restoring the oral 9 1155 11

Acknowledgements

Conflict of Interest Statement: None periosteal flap is only possible if an early diagnosis is made before the strength of the mandibular arch is weakened.

\author{
Acknowledgements
}




\section{References}

[1] - Marx RE. A new concept in the treatment of osteoradionecrosis. J Oral Maxillofac Surg. 1983;

41:351-7 (PMID: 6574217).

[2] - Wong JK, Wood RE, McLean M. Conservative management of osteoradionecrosis.Oral Surg Oral Med Oral Pathol Oral RadiolEndod. 1997; 84:16-21 (PMID: 9247943).

[3] - Lee M, Chin RY, Eslick GD, Sritharan N. Outcomes of microvascular free flap reconstruction for mandibular osteoradionecrosis : A systematic review. Journal of Cranio-Maxillo-facial Surgery.2015; 43:2026-2033 (DOI : 10.1016/j.jcms.2015.03.006).

[4] - Bettoni J, Olivetto M, Duisit J, Caula A, Bitar G, Lengele B et al. Role of « revascularization surgery $\gg$ in the management of mandibular osteoradionecrosis lesions : a report of 21 years of experience. Clin Otolaryngol 2018 dec 14. (DOI : 10.1111/coa.13272)

[5] - Martos-Fernandez M, Saez-Barba M, Lopez-Lopez J, Estrugo-Devesa A, Balibrea-DelCastillo JM, Bescos-Atin C. Pentoxifulline, Tocopherol, and Clodronate for the treatment of mandibular osteoradionecrosis: a systematic review. Oral Surg Oral Med Oral Pathol Oral Radiol 2018: 125:431-439 (DOI: 10.1016/j.oooo.2018.02.004)

[6] - Rice N, Polyzois I, Ekanayake K, Omer O, Stassen LFA. The management of osteoradionecrosis of jaws $\quad-$ A review.The Surgeon. 2015; 101-109 (DOI: 10.1016/j.surge.2014.07.003). 
[7] - D’Hauthuille C, Testelin S, Taha F, Bitar G, Devauchelle B. Part III : free periosteal flaps as a treatment for mandibular osteoradionecrosis. Rev Stomatol Chir Maxillofac 2009; 110: 3-7 (DOI: 10.1016/j.stomax.2008.07.007)

[8] - Yang GF, Chen PJ, Gao YZ, Liu XY, Li J, Jiang SX et al. Forearm free flap transplantation: a report of 56 cases. 1981. Br J Plast Surg 1997 ; 50: 162-5 (PMID: 9176001).

[9] - Guelinckx PJ, Boeckx WD, Fossion E, Gruwez JA. Scanning electron microscopy of irradiated recipient blood vessels in head and neck free flaps. Plast Reconstruct Surg $1984 ; 74: 217-226$ (PMID: 6463146)

[10] - Kelley P, Klebuc M, Hollier L. Complex midface reconstruction : maximizing contour and bone graft survival utilizing periosteal free flaps. J Craniofac Surg $2003 ; 14: 779-82$ (PMID: 14501347)

[11] - Roselli R, Muscatello L, Valdatta L, Pavan G, Spriano G. Mandibular reconstruction with frozen autologous mandibular bone and radial periosteal fasciocutaneous free flap : preliminary report. Ann Otol Rhinol Laryngol 2004 ;113:956-60 (DOI : 10.1177/000348940411301204)

[12] - Zhang C, Sun J, Zhu H, Xu L, Ji T, He Y et al. Microsurgical free flap reconstructions of the head and neck region : Shanghai experience of 34 years and 4640 flaps. Int J Oral Maxillofac Surg 2015 ;44:675-684 (DOI : 10.1016/j.ijom.2015.02.017)

[13] - Annane D, Depondt J, Aubert P et al. Hyperbaric oxygen therapy for radionecrosis of the jaw : a randomized, placebo-controlled, double-blind trial from the ORN96 study group. J Clin Oncol 2004; 22(24) :4893-900 (DOI: 10.1200/JCO.2004.09.006). 
214 [14] - Madrid C, Abarca M, Bouferrache K. Osteoradionecrosis : An update. Oral Oncology 2010; 2 21546 :471-474 (DOI : 10.1016/j.oraloncology.2010.03.017).

5

[15] - Delanian S, Chatel C, Porcher R et al. Complete restoration of refractory mandibular

osteoradionecrosis by prolonged treatment with a pentoxifylline-tocopherol-clodronate combination (PENTOCLO): a phase II trial. Int J Radiat Oncol Biol Phys 2011;80(3):832-9 (DOI: 10.1016/j.jijrobp.2010.03.029) 


\section{Captions to illustrations}

223

2 $22_{4}^{3}$ 5 225 7 226 10 227 12 1238 14 15
1229

Figure 1: Intraoperative photograph of the harvesting of the radial periosteal paddle (black arrow) achieved by dissecting the sensitive branches of the radial nerve (white arrow) which are retracted and protected by a Gillies hook on the tendon of the brachio-radial muscle. It is important to detach the subperiosteum carefully to avoid devascularization of the periosteal membrane secondary to decoupling from the radial vascular pedicle.

Figure 2: Intraoperative photographs before weaning of the periosteal forearm free flap and after releasing the touniquet to confirm (a) the viability of the skin paddle, and (b) the integrity and vascularization of the periosteal membrane. 
Figure 1
Click here to download high resolution image

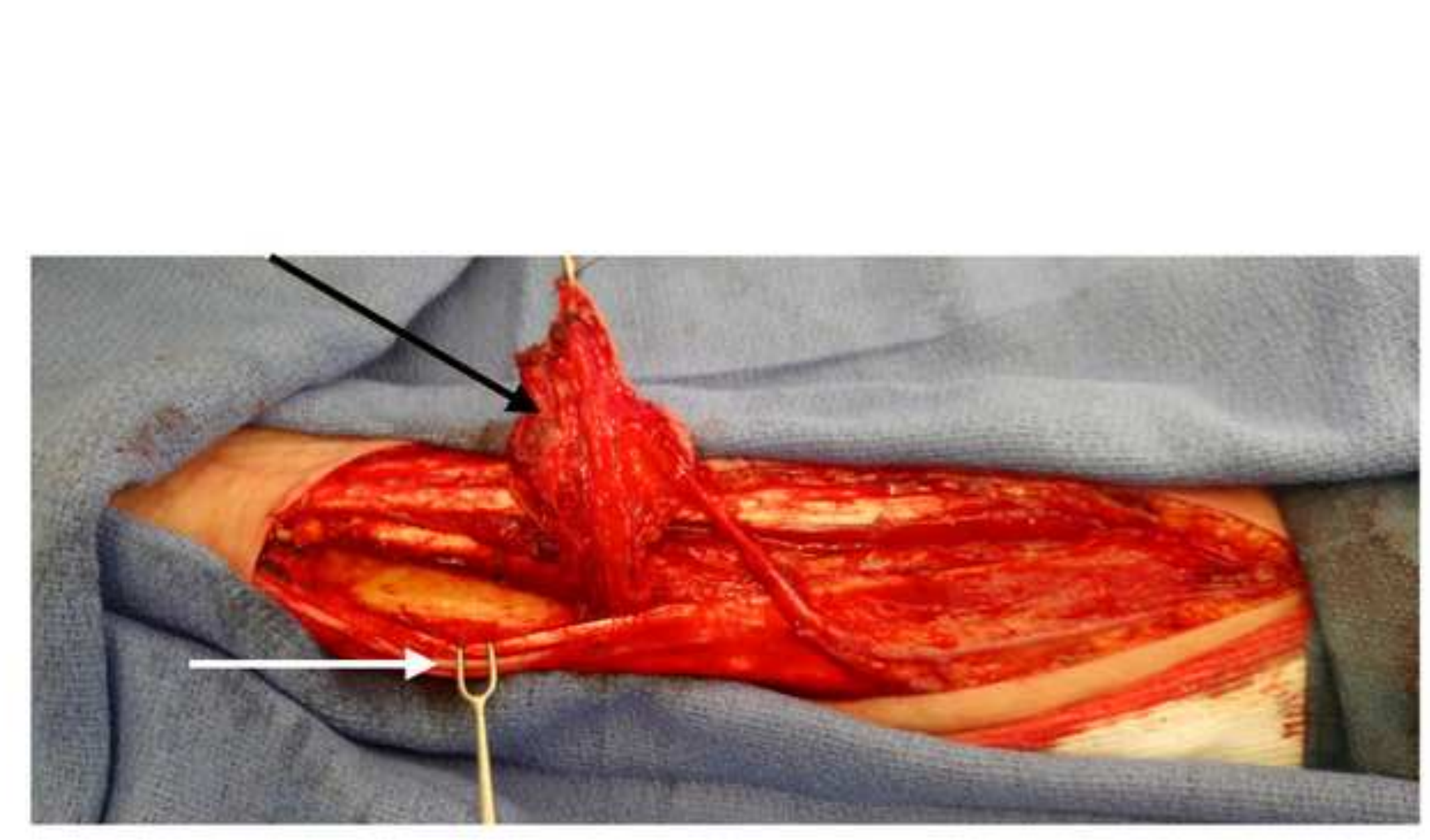

Figure 1

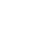

\author{
to download high resolution image
}
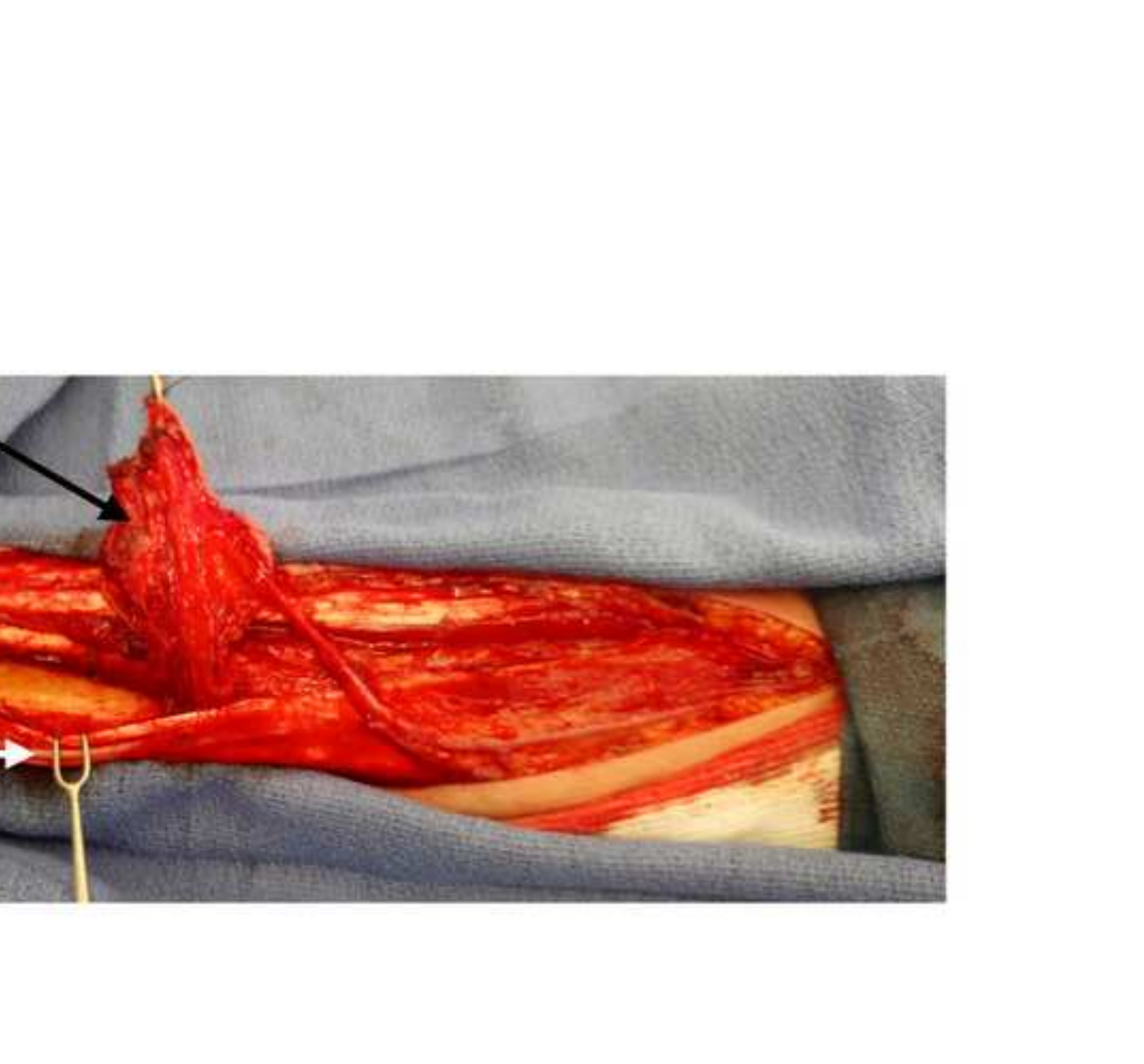


\section{Figure 2}

Click here to download high resolution image
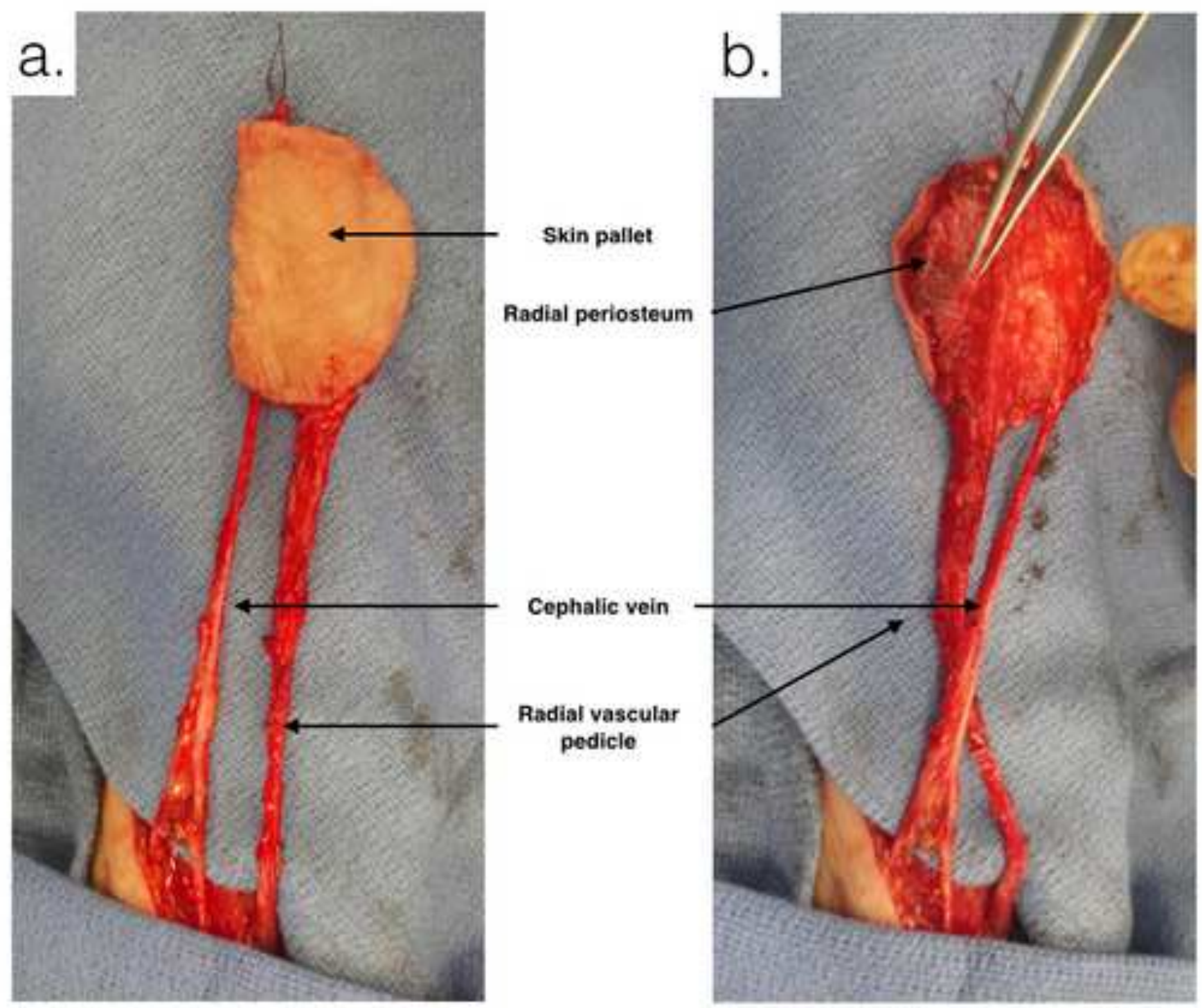